\title{
Alltagsmobilität in Kommunen zwischen Niederrhein und Ruhrgebiet. Aus welchen Gründen wird der ÖPNV nicht häufiger genutzt?
}

\author{
Eric Suder ${ }^{1}$ (D) Carmella Pfaffenbach ${ }^{1}$ \\ Eingegangen: 7. Mai 2020 / Überarbeitet: 8. Oktober 2020 / Angenommen: 14. Oktober 2020 / Online publiziert: 5. November 2020 \\ (c) Der/die Autor(en) 2020
}

\section{Zusammenfassung}

Die Alltagsmobilität ist in Deutschland stark mit der Nutzung des Pkw verbunden. Nachhaltigkeitsdebatten seit den 1980er-Jahren kritisieren jedoch die Dominanz des Pkw mit Verbrennungsmotor als Verkehrsmittel. Deshalb fordert die Wissenschaft verstärkt eine Verkehrswende zu klimaneutralen Antrieben sowie eine Verlagerung des Verkehrs auf öffentliche Verkehrsmittel, das Fahrrad und das Zufußgehen. In dieser Studie liegt der Fokus auf der Frage, welche Gründe aus Sicht der befragten Haushalte gegen die Nutzung des öffentlichen Personennahverkehrs (ÖPNV), der eine zentrale Säule dieser Transformation bildet, sprechen. Mit einem Fragebogen wurde die Alltagsmobilität von mehr als 1000 Bürgerinnen und Bürgern aus 4 Kommunen der westlichen Metropole Ruhr an der Schnittstelle zum Niederrhein untersucht. Die Analysen zeigen, dass zeitliche und infrastrukturelle Argumente am häufigsten vorgebracht werden, jedoch Unterschiede zwischen den Untersuchungsgemeinden und den befragten Haushalten bestehen. Um eine von Experten geforderte Verkehrsverlagerung vom Pkw auf andere Verkehrsmittel zu erreichen, müssen der nötige Zeitaufwand, die Zuverlässigkeit, die Flexibilität und auch das Preis-Leistungs-Verhältnis im öffentlichen Verkehr verbessert werden.

Schlüsselwörter Verkehrsmittelwahl · ÖPNV-Nutzung · Mobilitätswende · Quantitative Forschung · Empirische Sozialgeographie

\section{Everyday mobility in municipalities between the Lower Rhine and Ruhr areas. Why is public transport not used more frequently?}

\begin{abstract}
Everyday mobility in Germany is strongly related to the use of cars. However, sustainability debates since the 1980s have criticized the dominance of the car, with its internal combustion engine, as a means of transportation. Therefore, scientists are increasingly demanding a transition both to cars with climate-neutral engines and to a modal shift to public transportation, cycling, and walking. This study focuses on the reasons why the surveyed households are against the use of local public transport, which is a central element of this transformation. A questionnaire was used to investigate the everyday mobility of more than 1000 citizens from four municipalities in the western Rhine-Ruhr metropolitan area at the interface to the Lower Rhine region. The analyses show that time-based and infrastructural arguments are the most common but that there are differences between the study municipalities and the surveyed households. In order to achieve a modal shift from car to other means of transportation, as is demanded by experts, there is the need to improve time efficiency, reliability, flexibility, and the price-performance-ratio of public transportation.
\end{abstract}

Keywords Modal choice $\cdot$ Public transportation use $\cdot$ Modal shift · Quantitative research $\cdot$ Empirical social geography

Dr. Eric Suder

suder@geo.rwth-aachen.de

Prof. Dr. Carmella Pfaffenbach pfaffenbach@geo.rwth-aachen.de
1 Lehr- und Forschungsgebiet Kulturgeographie, Geographisches Institut, RWTH Aachen, Templergraben 55, 52056 Aachen, Deutschland 


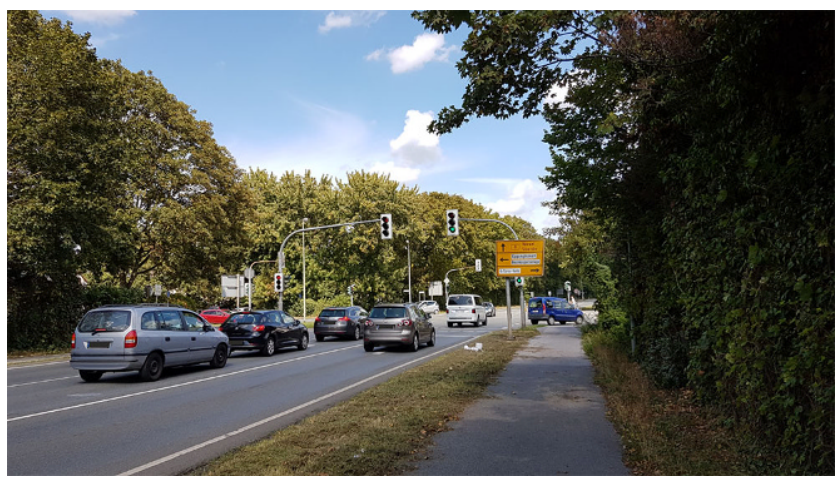

Abb. 1 Stark befahrene Straße in Dinslaken. (Foto: Autor)

\section{Einführung}

Der Pkw hat sich seit Ende der 1950er-Jahre in den westlichen Industrienationen zum dominierenden Verkehrsmittel entwickelt. Die Pkw-Dichte nahm in dieser Zeit in Deutschland insbesondere in den alten Bundesländern stark zu. Pro 1000 Einwohner waren 1955 weniger als 50 Pkw zugelassen. Ende der 1970er-Jahre waren es bereits mehr als 300 Pkw pro 1000 Einwohner (Lötscher et al. 2001, S. 62). Auch Städtebau und Stadtplanung haben sich in dieser Zeit an der Dominanz des Pkw orientiert und sie durch städtebauliche Leitbilder wie das der autogerechten Stadt (Reichow 1959) weiter gefördert. Edensor (2004) spricht in diesem Zusammenhang von Motorscapes, bei denen mobilitätsbezogene Infrastruktur auf das Automobil ausgerichtet ist. Derzeit sind in Deutschland rund 46,5 Mio. Pkw zugelassen. Dies entspricht einer Fahrzeugdichte von 563 Pkw pro 1000 Einwohner (KBA 2018, S. 67 ff.; vgl. Abb. 1).

Vor dem Hintergrund von Nachhaltigkeitsdebatten wird die Orientierung der Mobilität auf den Pkw seit den 1980erJahren zunehmend kritisiert. Begründet wird dies mit ökologischen Folgen der Verbrennungsmotoren wie der zunehmenden Feinstaubbelastung in Städten und dem Ausstoß von Treibhausgasen, die den Klimawandel forcieren. Aufgrund der Endlichkeit und Verteuerung der Ressource Öl wurde zudem mit steigenden Kosten für Mobilität argumentiert und damit auch der soziale Aspekt der Nachhaltigkeit von Automobilität infrage gestellt (vgl. Götz 2011, S. 329-330; Gudmundsson et al. 2016, S. 66 ff.).

Die in diesem Beitrag als Fallstudie ausgewählte Metropole Ruhr ist in besonderem Maße von Staus betroffen (ADAC 2019). Auf der anderen Seite ist das ÖPNVNetz in der Metropolregion - trotz aller Kritik (z. B. Überlastung, Verspätungen, fehlende Direktverbindungen; vgl. Baltes et al. 2008, S. 76) - vergleichsweise gut ausgebaut und verbindet alle Teile der polyzentrisch gegliederten Region miteinander. Dies macht ÖPNV-Nutzung an sich zu einer guten Alternative zum Pkw. Jedoch gibt es in der Metropole Ruhr große Unterschiede, was die ÖPNV-Erschlie- ßung einerseits und die Bevölkerungs- und Pkw-Dichte und damit die Staugefahr andererseits betrifft. An dieser Stelle setzt der vorliegende Beitrag mit der Frage an, weshalb der ÖPNV nicht häufiger genutzt wird. Die Motive für die Nichtnutzung werden dabei aus der Perspektive der Bewohner/innen von 4 Kommunen an der Schnittstelle von Niederrhein und Ruhrgebiet beleuchtet.

\section{Alltagsmobilität im räumlichen und gesellschaftlichen Kontext}

In den letzten Jahrzehnten sind in Deutschland sowohl der Motorisierungsgrad als auch die Verkehrsleistung kontinuierlich angestiegen (BMVI 2018a). Aktuelle Trends zeigen jedoch eine geringere Pkw-Nutzung bei jungen Erwachsenen (ifmo 2011). Zwar kommt auch die Befragung Mobilität in Deutschland 2017 zu dem Ergebnis, dass junge Erwachsene in größeren Städten weniger Pkw-orientiert sind als es frühere Generationen waren, gleichzeitig geht als Erkenntnis hervor, dass ,trotz einer Steigerung der Nutzungsanteile von öffentlichen Verkehrsmitteln und des Fahrrads ... das Auto nach wie vor das dominante Verkehrsmittel der Alltagsmobilität in Deutschland" (BMVI 2018a, S. 3) ist. Manderscheid führt die Dominanz des Autos auf die Identifikation der Gesellschaft mit dem Auto zurück und argumentiert, dass die „Automobilität ein fundamentaler Bestandteil ...der Subjektivierungsformen gegenwärtiger Gesellschaften“ (2013, S. 105) sei, die seit „einem bestimmten Verbreitungs- und Nutzungsgrad privater Autos ... hegemonial in dem Sinne [wurde], dass das Leben in westlichen Gesellschaften automobile Subjekte quasi voraussetzte“ (2013, S. 108). Nichtsdestotrotz wird die Alltagsmobilität durch ein komplexes Zusammenspiel u. a. von soziodemografischen, räumlichen und soziopsychologischen Faktoren geprägt (vgl. De Witte et al. 2013; Hunecke 2015; Hunecke und Schweer 2006).

Im Zusammenhang mit den bereits erwähnten Nachhaltigkeitsdebatten und den negativen Folgen der Automobilität wird bereits seit den 1990er-Jahren (Hesse 1993), spätestens aber seit der Klimakonferenz in Paris 2015 politisch eine Verkehrswende gefordert, die die Klimaneutralität des Verkehrs erreichen soll (Rudolph et al. 2017, S. 9). Neben der Dekarbonisierung, also einer Energiewende hin zu emissionsfreien Antrieben, basiert die Verkehrswende auf einer Mobilitätswende, die die Vermeidung und Verlagerung des Verkehrs vom Auto zu öffentlichem Verkehr, Fahrrad und Zufußgehen vorsieht (Agora Verkehrswende 2017). Zum Stand der Verkehrswende resümiert Mobilität in Deutschland 2017, dass sie zwar ,in Ansätzen sichtbar, aber bei weitem nicht vollzogen" (Follmer und Gruschwitz 2018, S. 7) sei. 


\section{Methodisches Vorgehen}

Die vorliegende Studie beruht auf einer schriftlichen standardisierten Befragung, in der neben Fragen zum Mobilitätsverhalten und den Hintergründen der Verkehrsmittelwahl ein Wegeprotokoll für einen vorgegebenen Tag des Ergebungszeitraums enthalten war. Die Erhebung fand im Frühjahr 2018 an 5 Erhebungstagen statt. Insgesamt konnten 1062 Personen befragt werden. Es ist darauf hinzuweisen, dass es sich bei dieser Studie nicht um eine repräsentative Umfrage handelt, da die Auswahl der Befragten keiner reinen Zufallsstichprobe unterlag. Die Teilnehmerinnen und Teilnehmer wurden durch Haustürbefragung und Ansprache auf öffentlichen Plätzen und in öffentlichen Einrichtungen gewonnen. Durch verschiedene Erhebungstage und -zeiten sowie der genannten Vorgehensweise vor Ort wurde versucht, einen strukturellen Ausschluss bestimmter Personengruppen zu minimieren (z.B. Berufstätige durch Erhebung in frühen Abendstunden und am Wochenende). Aufgrund dessen und durch die relativ große Stichprobe konnte insgesamt ein aussagekräftiges Ergebnis erzielt werden.

Die Auswahl des Untersuchungsgebiets folgte verschiedenen Kriterien. Zum einen sollten zwischen den ausgewählten Kommunen sowohl die Einwohnerzahlen stark voneinander abweichen als auch ein großes Einwohnerdichtegefälle vorliegen, um dem heterogenen Raum Metropole Ruhr gerecht zu werden. Die Metropolen im Zentrum der Metropole Ruhr wurden dabei bewusst nicht ausgewählt, da sich die Studie vor allem mit der Nichtnutzung des ÖPNV befasst und im Kern der Metropole Ruhr ein vergleichsweise hoher ÖPNV-Anteil erwartet wurde. Zum anderen sollten die Untersuchungskommunen räumlich und infrastrukturell eng miteinander verbunden sein, um externe Einflussfaktoren, wie z.B. abweichende ÖPNVTarifgebiete, zu minimieren. Als Untersuchungskommunen wurden Oberhausen, Dinslaken, Voerde und Hünxe ausgewählt, die im Westen der Metropole Ruhr unmittelbar aneinandergrenzen (vgl. Tab. 1). Gleichzeitig sollten die soziodemographischen Rahmenbedingungen vergleichbar sein. Dabei wurde auf eine ähnliche Altersstruktur und eine relativ stabile Bevölkerungsentwicklung geachtet, sodass altindustrialisierte Kommunen mit starkem Bevölkerungsrückgang ausgeschlossen wurden.

\section{Alltagsmobilität in Kommunen zwischen Niederrhein und Ruhrgebiet}

Von den 1062 Befragten füllten 672 das Wegeprotokoll aus und gaben bis zu 7 Wege für einen Donnerstag im Erhebungszeitraum an. Insgesamt gaben die Befragten 2182 Wege zu Protokoll. Somit wurden im Durchschnitt 3,2 Wege pro Tag angegeben. Von den protokollierten Wegen waren 197 (9\%) multimodal, wurden also mit mehreren Verkehrsmitteln zurückgelegt.

Abb. 2 zeigt den Modal Split der Untersuchungskommunen im Vergleich zu Kommunen gleichen Raumtyps aus der Studie Mobilität in Deutschland 2017. Trotz eines vergleichsweise hohen ÖPNV-Anteils ist auch in der Großstadt Oberhausen der Pkw mit Abstand das dominante Verkehrsmittel. Neben einer überdurchschnittlichen Pkw-Nutzung in allen Kommunen werden in Oberhausen Wege seltener zu Fuß und mit dem Fahrrad zurückgelegt, was sich mit der Stadtstruktur begründen lässt, da unter anderem aufgrund mehrerer Bahntrassen im Stadtgebiet, die nur an wenigen viel befahrenen Unterführungen gequert werden können, lange Wege zwischen Zielen entstehen können. In Dinslaken und Voerde ist der Anteil der Pkw-Nutzung auf Kosten von nichtmotorisierten Verkehrsmitteln und dem ÖPNV höher und in Hünxe wird der ÖPNV fast gar nicht genutzt.

Unabhängig vom Wegeprotokoll gaben in jeder Kommune mehr als $60 \%$ der Befragten an, den Pkw täglich zu nutzen. Mindestens wöchentlich wird das Auto von $90 \%$ der Befragten in Oberhausen und Dinslaken sowie von $95 \%$ der Befragten in Voerde und Hünxe genutzt. Im Gegensatz dazu gaben etwa $30 \%$ der Oberhausener an, den Bus mindestens wöchentlich zu nutzen, aber nur $14 \%$ der Dinslakener, $7 \%$ der Voerder und $4 \%$ der Hünxer Befragten. Auch die Nutzung alternativer Verkehrsmittel wurde erhoben. Zwar nutzen immerhin 7\% der Befragten derzeit ein Elektrofahrrad, jedoch fällt die aktuelle Nutzung von E-/ Hybrid-Pkw, Car-/Bikesharing und Bürgerbussen mit Werten von weniger als $1 \%$ sehr gering aus. Daher setzt der

Tab. 1 Übersicht der Untersuchungskommunen. (Daten: IT.NRW 2018)

\begin{tabular}{llll}
\hline & Oberhausen & Dinslaken & Voerde \\
\hline Gemeindetyp & Kleine Großstadt & Große Mittelstadt & Kleine Mittelstadt \\
Einwohnerzahl & 211.422 & 67.489 & 36.268 \\
Bevölkerungsdichte (in Einwohner/km²) & 2742 & 1416 & 678 \\
Pkw-Dichte (je 1000 Einwohner) & 517 & 598 & 609 \\
Wichtige Straßen & A3, A42, A516 & A3, B8 & A3, B8 \\
ÖPNV-Angebot & Zug (4 Bahnhöfe), & Zug (1 Bahnhof), & Zug (2 Bahnhöfe), \\
& Straßenbahn, Bus & Straßenbahn, Bus & Bus \\
\hline
\end{tabular}


Abb. 2 Modal Split in der Kernwoche im Vergleich zu Kommunen gleichen Raumtyps. (Eigene Erhebung, BMVI 2018b)

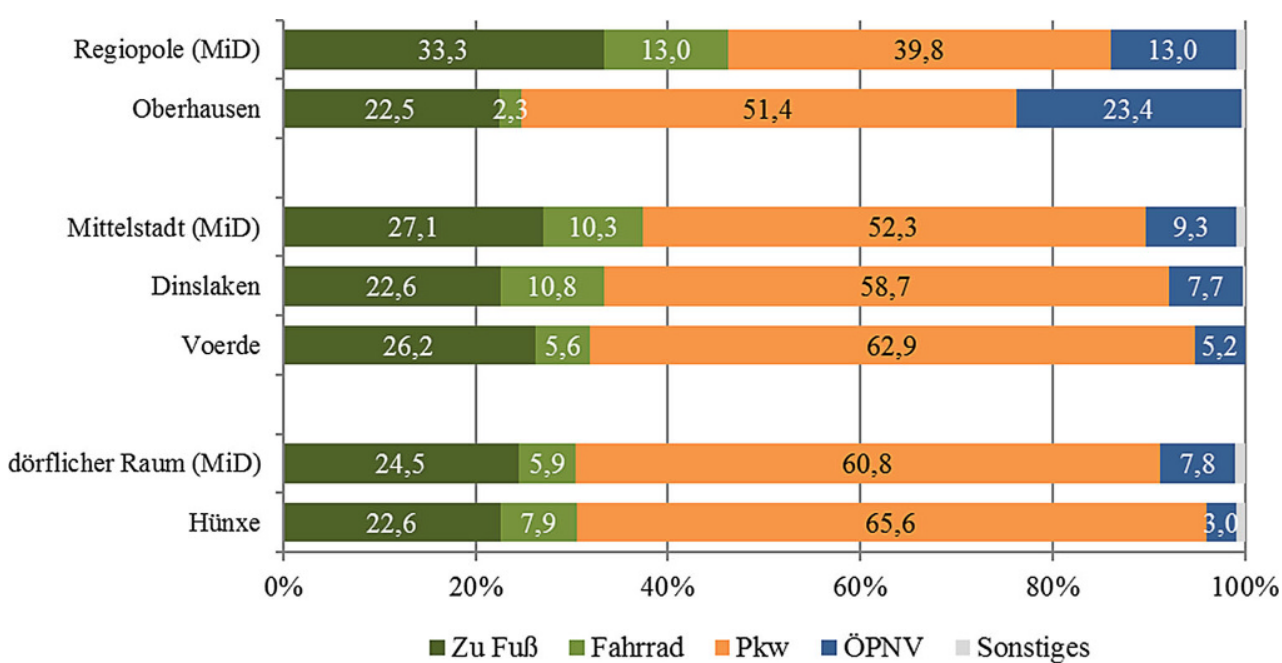

Artikel den Fokus auf die (Nicht-)Nutzung des ÖPNV, der als eine der wichtigen Säulen der Verkehrswende zu sehen ist.

\section{Gründe für die Nichtnutzung des ÖPNV im räumlichen Kontext}

Wie bereits in der Einleitung erwähnt, stellt die Metropole Ruhr ein Untersuchungsgebiet dar, das ein in weiten Teilen gut ausgebautes ÖPNV-Netz besitzt, gleichzeitig aber in besonderem Maße von Staus betroffen ist. Dies führt in dieser Studie zu der Frage, weshalb sich Personen - abseits der großen Metropolen im Zentrum der Metropole Ruhr - trotz der genannten Gründe für den Pkw und gegen den ÖPNV als Verkehrsmittel entscheiden. Dazu wurde im Fragebogen eine offene Frage zu den Hintergründen der Nichtnutzung des ÖPNV gestellt, sofern dieser nicht regelmäßig genutzt wird. Eine maximale Nennung von 3 Gründen war möglich. Negative Korrelationen zwischen der Nutzung von Pkw und
Bus $(-0,399 * *)$ bzw. Zug $(-0,312 * *)$ werden auch hier in vielen Bereichen bestätigt, da viele Gründe, die gegen den ÖPNV sprechen, gleichzeitig Gründe für die Nutzung des Pkw darstellen (z. B. Flexibilität, Komfort, 24h-Verfügbarkeit).

Etwa $62 \%$ aller Befragten gaben Gründe an, die aus ihrer Sicht gegen die Nutzung des ÖPNV sprechen. Der Anteil variiert nach Wohnort. In Oberhausen nannten lediglich $56 \%$ Gründe gegen eine regelmäßige ÖPNV-Nutzung, mit abnehmender Einwohnerzahl steigt dieser Wert bis auf $69 \%$ in Hünxe. Insgesamt wurden zeitliche und infrastrukturelle Aspekte sowie fehlender Komfort, mangelnde Flexibilität und als zu hoch wahrgenommene Kosten (im Vergleich zum Pkw) am häufigsten genannt (vgl. Tab. 2).

Aufgrund eines ÖPNV-Angebots, das sich werktags auf den Busverkehr im 2-Stunden-Takt beschränkt und am Wochenende zum Teil gar nicht vorhanden ist (vgl. Abb. 3), ist das eingeschränkte Angebot an öffentlicher Verkehrsinfrastruktur (Zugänglichkeit, Erreichbarkeit) mit deutlichem Abstand (59\% aller Nennungen) der bedeutendste Faktor

Tab. 2 Übersicht der genannten Gründe. (Eigene Erhebung)

\begin{tabular}{lll}
\hline Kategorie & Wert (\%) & Erläuterung \\
\hline Zeitliche Faktoren & 26,6 & Reisezeit des ÖPNV (im Vergleich zum Pkw) \\
Infrastruktur & 25,4 & Fehlende Direktverbindungen, Entfernung zur Haltestelle \\
Komfort & 24,3 & Überfüllung, fehlende Transportmöglichkeit, Bequemlichkeit, Mitreisende, Sauberkeit \\
Flexibilität & 23,4 & Fehlende Flexibilität, Abhängigkeit vom Fahrplan \\
Kosten & 22,4 & Preis-Leistungs-Verhältnis, (wahrgenommene) Kosten im Vergleich zum Pkw \\
Nutzung von Alternativen & 14,1 & „Pkw-Besitz“, Vorteile des Pkw (Schnelligkeit, Komfort, Flexibilität, steht vor der Tür) \\
Taktung & 13,4 & Generelle Fahrthäufigkeit, zu lange/kurze Umsteigezeit, Bedienzeit (abends, am Wochenende) \\
Zuverlässigkeit & 12,4 & Unpünktlichkeit \& Verspätungen \\
Kein Bedarf & 7,3 & Fußläufige Erreichbarkeit (Wohnortlage) \\
Sicherheitsbedenken & 5,0 & Unsicherheitsgefühl an Haltestellen oder im Bus \\
Eigene Gesundheit & 3,9 & Körperliche Einschränkungen, fehlende Barrierefreiheit \\
Fehlende Kenntnisse & 1,9 & Kompliziertes Tarifsystem \\
\hline
\end{tabular}




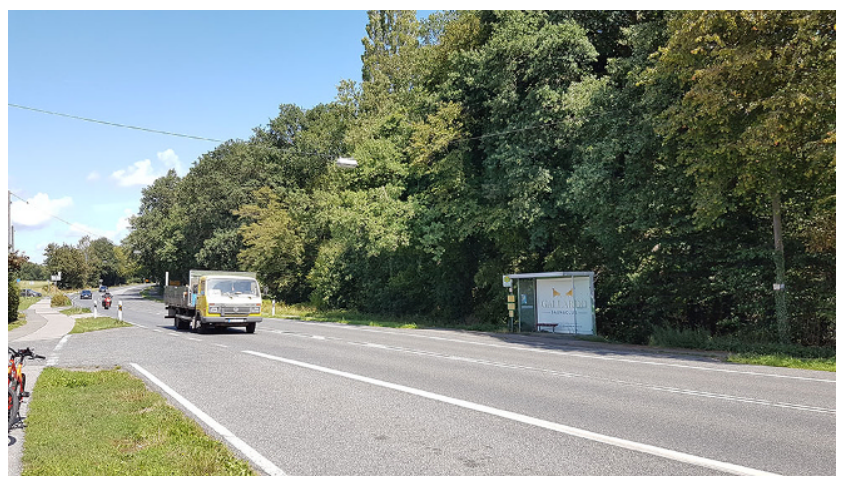

Abb. 3 Verlassene Haltestelle in Hünxe-Bruckhausen. (Foto: Autor)

der Nichtnutzung des ÖPNV in Hünxe (vgl. Abb. 4). Damit in Verbindung stehen auch weitere Faktoren wie die eingeschränkte Flexibilität (18\%) sowie die Unzufriedenheit mit dem Preis-Leistungs-Verhältnis (20\%).

Im Vergleich zu Hünxe ist die nötige Verkehrsinfrastruktur in Voerde zwar gegeben, jedoch wurden andere Faktoren fast gleich häufig von mehr als einem Fünftel aller Befragten genannt. Dazu zählen die fehlende Flexibilität (23\%), das Vorhandensein anderer Verkehrsmittel respektive des Pkw (22\%) und die geringe Taktung des ÖPNV (22\%).

Obwohl auch in Dinslaken zahlreiche Mobilitätsoptionen vorhanden sind, wird die Infrastruktur überdurchschnittlich oft als Grund für die Nichtnutzung des ÖPNV genannt $(35 \%)$. Insbesondere wird von einer ,schlechten Anbindung" gesprochen. Darüber hinaus nannten je ein Viertel der Befragten zeitliche Gründe und fehlenden Komfort und dabei vor allem die eigene Bequemlichkeit.

In Oberhausen wird ebenfalls der Komfort kritisiert, hier jedoch aufgrund von überfüllten Bussen und Straßenbahnen sowie mangelnder Sauberkeit. Wird der ÖPNV nicht regelmäßig genutzt, sind in Oberhausen zudem zeitliche Gründe $(29 \%)$, fehlende Flexibilität $(25 \%)$ und zu hohe Kosten (25\%) entscheidend.

\section{Gründe für die Nichtnutzung des ÖPNV mit Blick auf weitere Indikatoren}

Bei der Betrachtung verschiedener Haushaltstypen zeigen sich ebenfalls Unterschiede in den Begründungen (vgl. Abb. 5). Es wurden 4 Haushaltstypen verglichen: Haushalte mit Kindern unter 10 Jahren (,jüngere Familien“), Haushalte mit Kindern ausschließlich zwischen 10 und 17 Jahren (,ältere Familien“), kinderlose Haushalte zwischen 20 und 49 Jahren sowie Senioren-Haushalte über 65 Jahren. Insgesamt gaben rund zwei Drittel der kinderlosen Haushalte und Haushalte mit Kindern Gründe an, nicht regelmäßig mit dem ÖPNV zu fahren, jedoch lediglich $54 \%$ der Senioren. Sowohl Familien mit jüngeren als auch älteren Kindern nannten besonders häufig infrastrukturelle (33\% bzw. $32 \%$ ) und zeitliche Gründe ( $29 \%$ bzw. 24\%). Hinzu kommt als Argument jüngerer Familien die fehlende Flexibilität (33\%). Kinderlose Haushalte gaben unabhängig von der Kommune zu hohe Kosten im ÖPNV (36\%) als Hauptgrund für die seltene Nutzung des Nahverkehrs an, während es bei Senioren anteilig meist die Verkehrsinfrastruktur $(39 \%)$ und die Nutzung von Alternativen (23\%) waren. Zeitliche Faktoren und Kosten spielen dagegen bei Senioren eine geringere Rolle.

Während sich keinerlei genderspezifische Korrelationen bei der Argumentation für die Nichtnutzung des ÖPNV feststellen lassen, spielt das Alter der Befragten durchaus eine Rolle. Insbesondere finanzielle Aspekte $(0,159 * *)$ und mangelnde Zuverlässigkeit $(0,172 * *)$ wurden etwas häufiger von älteren Personen genannt.

Haushalte mit einem höheren Einkommen argumentierten wiederum öfter mit mangelnder Infrastruktur $\left(0,108^{*}\right)$ und Flexibilität $(0,139 * *)$ sowie fehlendem Komfort $(0,104 *)$, jedoch überaschenderweise seltener mit der Nutzung von Alternativen $\left(-0,178^{* *}\right)$. Eine mögliche Erklärung wäre, dass Haushalte mit niedrigerem Einkommen zwar seltener den Pkw im Alltag nutzen $(-0,244 * *)$, jedoch häufiger Zufußgehen $\left(0,120^{* *}\right)$ und insgesamt auch häufiger mit dem ÖPNV mobil sind.

\section{Fazit}

Die Ergebnisse dieser Studie zeigen, dass aus Sicht der Befragten zeitliche und infrastrukturelle Aspekte sowie fehlender Komfort, mangelnde Flexibilität und zu hohe Kosten gegen die Nutzung des ÖPNV sprechen. Während in anderen Studien (z. B. Beirão und Sarsfield Cabral 2007) insbesondere Schnelligkeit, Flexibilität und Zuverlässigkeit des ÖPNV im Vergleich zu anderen Verkehrsmitteln bemängelt wurden, wurde in unserer Befragung, die zumindest teilweise in einer Kleinstadt erfolgte, auf infrastrukturelle Defizite (fehlende Verbindungen oder zu seltene Taktung) und finanzielle Gründe (hohe Ticketkosten) verwiesen. Es zeigte sich, dass sich insbesondere jüngere Familien bei zeitlichen und infrastrukturellen Aspekten sowie bei Flexibilität mit Verweis auf spezifische Anforderungen und Einschränkungen, die sie wegen ihrer kleinen Kinder haben, kritischer äußerten als beispielsweise Senioren. Die Ergebnisse der Studie machen zudem deutlich, dass die Bewertung des ÖPNV - explizit und implizit - im Vergleich mit dem vermeintlich attraktiveren Pkw vorgenommen wird. Somit konkurriert der ÖPNV auf individueller Ebene mit den Vorteilen der Pkw-Mobilität (Schnelligkeit, Verfügbarkeit, Flexibilität, Komfort).

Für die angestrebte Verkehrswende bedeutet dies, dass nicht nur alternative Antriebe etabliert werden müssen, son- 
Abb. 4 Häufigste Gründe für die Nichtnutzung des ÖPNV nach Wohnort (in \% aller Nennungen). (Eigene Erhebung)

Abb. 5 Häufigste Gründe für die Nichtnutzung des ÖPNV nach Haushaltstyp (in \% aller Nennungen). (Eigene Erhebung)
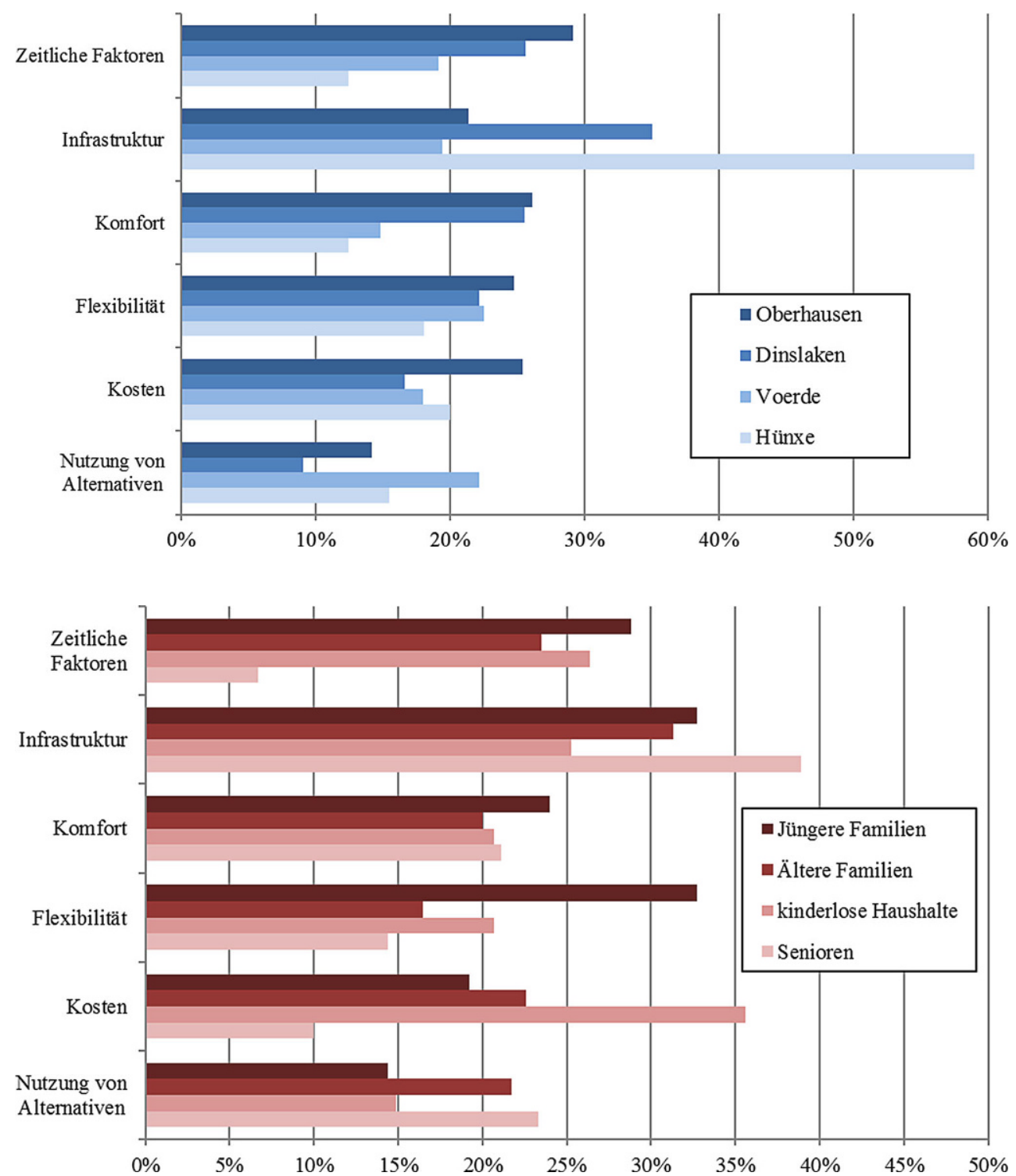

dern auch Anreize zu schaffen sind, damit Pkw-Nutzer auf andere Verkehrsmittel umsteigen. Neben dem Fahrradfahren und Zufußgehen sowie der Nutzung von Sharing-Angeboten bietet vor allem der ÖPNV ein großes Potenzial für die Mobilitätswende, zumindest was größere Distanzen und täglich wiederkehrende Wege, z. B. zum Arbeitsplatz, betrifft. Um für die Befragten attraktiv(er) zu sein, müsste ein zukünftiges ÖPNV-System in der Untersuchungsregion jedoch einige Voraussetzungen erfüllen. Erstens müsste es aus Sicht der Befragten zeitlich konkurrenzfähig gegenüber anderen Verkehrsmitteln sein. Dies betrifft nicht nur den reinen Fahrweg, sondern schließt Warte- und Umsteigezeiten mit ein, die häufig als länger wahrgenommen werden als die Parkplatzsuche mit dem Pkw. Zweitens müsste das System zeitlich und räumlich flexibel sein. Eine geringe Taktung mit begrenzter Anzahl von Busverbindungen wurde von den Befragten in den klein- und mittelstädtischen
Untersuchungsgemeinden oftmals als Ausschlusskriterium für dessen Nutzung genannt. Hier wären bedarfsorientierte Mobilitätskonzepte nötig, die sich nicht an einem Fahr- und Linienplan orientieren, sondern je nach Bedarf zur Verfügung stehen. Und drittens müsste eine Erhöhung der Qualität des ÖPNV für die Nutzer finanzierbar bleiben. $20 \%$ der Befragten gaben an, dass sie die Preise des ÖPNV als zu hoch empfinden. Dabei ist sowohl der (subjektive) Vergleich mit dem Pkw gemeint als auch das Preis-LeistungsVerhältnis. Unter Leistung haben die Befragten auch die Zuverlässigkeit des ÖPNV häufig genannt.

Um die Attraktivität des ÖPNV in der Untersuchungsregion zu erhöhen, sind also - den Untersuchungsergebnissen zufolge - sowohl eine Verbesserung des Preis-LeistungsVerhältnisses als auch ein zusätzlicher Ausbau der ÖPNVInfrastruktur erforderlich. Da die Ergebnisse der Untersuchung insbesondere gezeigt haben, dass die Bewertung des 
ÖPNV im direkten Vergleich mit dem Pkw erfolgt, könnte eine Steigerung der Attraktivität des ÖPNV aber auch durch eine Verringerung der derzeitigen Pkw-Attraktivität erreicht werden (z. B. durch höhere Kosten für das Fahren und innerstädtische Parken von Pkw oder durch die Einführung von Pkw-freien Innenstädten).

Zudem bieten alternative Verkehrsmittel und Antriebe Potenzial für die angestrebte Mobilitätswende. So gaben $55 \%$ der Befragten an, sich vorstellen zu können zukünftig Elektro- oder Hybrid-Pkw zu nutzen, bei E-Fahrrädern waren es immerhin $43 \%$ und bei Bürgerbussen und autonom fahrenden Fahrzeugen 23\%. Dabei ist die Bereitschaft, in Zukunft E-/Hybrid-Pkw und autonome Fahrzeuge zu nutzen, erwartungsgemäß bei den jüngeren Befragten signifikant höher.

Danksagung Wir möchten an dieser Stelle den Studierenden des Projektseminars Angewandte Stadtgeographie für ihren Einsatz während des Geländepraktikums sehr herzlich danken.

Funding Open Access funding enabled and organized by Projekt DEAL.

Open Access Dieser Artikel wird unter der Creative Commons Namensnennung 4.0 International Lizenz veröffentlicht, welche die Nutzung, Vervielfältigung, Bearbeitung, Verbreitung und Wiedergabe in jeglichem Medium und Format erlaubt, sofern Sie den/die ursprünglichen Autor(en) und die Quelle ordnungsgemäß nennen, einen Link zur Creative Commons Lizenz beifügen und angeben, ob Änderungen vorgenommen wurden.

Die in diesem Artikel enthaltenen Bilder und sonstiges Drittmaterial unterliegen ebenfalls der genannten Creative Commons Lizenz, sofern sich aus der Abbildungslegende nichts anderes ergibt. Sofern das betreffende Material nicht unter der genannten Creative Commons Lizenz steht und die betreffende Handlung nicht nach gesetzlichen Vorschriften erlaubt ist, ist für die oben aufgeführten Weiterverwendungen des Materials die Einwilligung des jeweiligen Rechteinhabers einzuholen.

Weitere Details zur Lizenz entnehmen Sie bitte der Lizenzinformation auf http://creativecommons.org/licenses/by/4.0/deed.de.

\section{Literatur}

ADAC - Allgemeiner Deutscher Automobil-Club (Hrsg) (2019) ADAC Staubilanz 2018: 486.000 Kilometer Stau in NRW! https://presse.adac.de/regionalclubs/nordrhein-westfalen/adacstaubilanz-nrw-2018.html. Zugegriffen: 1. Apr. 2020

Agora Verkehrswende (2017) Mit der Verkehrswende die Mobilität von morgen sichern. 12 Thesen zur Verkehrswende. Agora Verkehrswende, Berlin

Baltes H, Drobek S, Griesenbrock K, Schönharting J (2008) Mobilität in der Metropole Ruhr im Vergleich zu anderen Metropolen. Analysen, Leitbild, Konzepte, Maßnahmen. Essen. http:// trc-transportation.com/cms/images/dokumente/MetropoleRuhrStudie_Endbericht_kurz.pdf
Beirão G, Sarsfield Cabral J (2007) Understanding attitudes towards public transport and private car: a qualitative study. Transp Policy 14:478-489

BMVI - Bundesministerium für Verkehr und digitale Infrastruktur (Hrsg) (2018a) Mobilität in Deutschland 2017. Ergebnisbericht. BMVI - Bundesministerium für Verkehr und digitale Infrastruktur (Hrsg), Bonn

BMVI - Bundesministerium für Verkehr und digitale Infrastruktur (Hrsg) (2018b) Tabellentool Mobilität in Tabellen (MiT 2017) der Erhebung Mobilität in Deutschland. https://test1.q-dot.de/ mit. Zugegriffen: 1. Apr. 2020

De Witte A, Hollevoet J, Dobruszkes F, Hubert M, Macharis C (2013) Linking modal choice to motility: a comprehensive review. Transp Res A 49:329-341. https://doi.org/10.1016/j.tra.2013.01.009

Edensor T (2004) Automobility and national identity: representation, geography and driving practice. Theory Cult Soc 21(5):101-120. https://doi.org/10.1177/0263276404046063

Follmer R, Gruschwitz D (2018) Mobilität in Deutschland - MiD Kurzreport. Bonn, Berlin (Studie von infas, DLR, IVT und infas 360 im Auftrag des Bundesministers für Verkehr und digitale Infrastruktur)

Götz K (2011) Nachhaltige Mobilität. In: Groß M (Hrsg) Handbuch Umweltsoziologie. Springer, Wiesbaden, S 325-347

Gudmundsson H, Hall R, Marsden G, Zietsman J (2016) Sustainable Transportation. Indicators, Frameworks, and Performance Management. Springer Texts in Business and Economics, Heidelberg

Hesse M (1993) Verkehrswende. Ökologisch-ökonomische Perspektiven für Stadt und Region. Metropolis, Marburg

Hunecke M (2015) Mobilitätsverhalten verstehen und verändern. Psychologische Beiträge zur interdisziplinären Mobilitätsforschung. Springer, Wiesbaden

Hunecke M, Schweer I (2006) Einflussfaktoren der Alltagsmobilität Das Zusammenwirken von Raum, Verkehrsinfrastruktur, Lebensstil und Mobilitätseinstellungen. In: Beckmann K, Hesse M, HolzRau C, Hunecke M (Hrsg) StadtLeben - Wohnen, Mobilität und Lebensstil. VS, Wiesbaden, S 148-166

ifmo - Institut für Mobilitätsforschung (Hrsg) (2011) Mobilität junger Menschen im Wandel - multimodaler und weiblicher. ifmo, München

IT.NRW (2018) Kommunalprofile. Stand 29.08.2018. www.it.nrw/ kommunalprofile.. Zugegriffen: 1. Apr. 2020

KBA - Kraftfahrt-Bundesamt (2018) Fahrzeugzulassungen (FZ). Bestand an Nutzfahrzeugen, Kraftfahrzeugen insgesamt und Kraftfahrzeuganhängern nach technischen Daten. 1. Januar 2018. FZ 25. KBA, Flensburg

Lötscher L, Mayer O, Monheim R (2001) Entwicklung der privaten Motorisierung. In: Institut für Länderkunde (Hrsg) Nationalatlas Bundesrepublik Deutschland. Verkehr und Kommunikation. Nationalatlas, 9. Springer, Heidelberg, Berlin, S 62-63

Manderscheid K (2013) Automobile Subjekte. In: Scheiner J, Blotevogel H-H, Frank S, Holz-Rau C, Schuster N (Hrsg) Mobilitäten und Immobilitäten. Blaue Reihe - Dortmunder Beiträge zur Raumplanung. Klartext, Essen, S 105-120

Reichow HB (1959) Die autogerechte Stadt. Ein Weg aus dem Verkehrs-Chaos. Otto Meier, Ravensburg

Rudolph F, Koska T, Schneider C (2017) Verkehrswende in Deutschland. Der Weg zu CO 2 -freier Mobilität bis 2035. Wuppertal Institut für Klima, Umwelt, Energie, Wuppertal 